\title{
Determinants of disability in osteoarthritis of the
} knee

\author{
T E McAlindon, C Cooper, J R Kirwan, P A Dieppe
}

\begin{abstract}
Objectives To evaluate the influences of radiographic severity, quadriceps strength, knee pain, age, and gender on functional ability in patients with osteoarthritis of the knee.

Methods Equal numbers of knee pain positive and negative respondents to a survey of registrants aged more than 55 years at a general practice were invited to attend for knee radiographs and quadriceps femoris isometric strength estimations. Disability was measured using the Stanford Health Assessment Questionnaire.
\end{abstract}

Results Complete data were available on 70 men (mean age 72.7 years) and 89 women (mean age 68.1 years); $44 \%$ reported knee pain, $48 \%$ had radiographic features of osteoarthritis, and $32 \%$ reported some degree of disability. Significant correlations were observed between disability and radiographic score, quadriceps strength, and knee pain. Logistic regression analysis, however, showed significant independent contributions from quadriceps strength (odds ratio $0.84 \mathrm{kgF}$ ), knee pain (odds ratio 1.67 ), and age (odds ratio 1.06 per year) only; the radiographic score had no influence on the model. These results were not influenced by confining the analysis to the group with radiographic features of osteoarthritis.

Conclusions Quadriceps strength, knee pain, and age are more important determinants of functional impairment in elderly subjects than the severity of knee osteoarthritis as assessed radiographically. Strategies designed to optimise muscle strength may have the potential to reduce a vast burden of disability, dependency, and cost.

(Ann Rheum Dis 1993; 52: 258-262)

Rheumatology Unit, Bristol Royal Infirmary, Bristol BS2 8HW, United Kingdom $T$ E McAlindon C Cooper J R Kirwan

P A Dieppe Correspondence to: Rheumatology Department, St Thomas's Hospital, London SE1 7EH,

Accepted for publicatio 3 November 1992

Physical disability is now a major public health problem in elderly subjects. ${ }^{1}$ Problems with locomotion are the most common disorders, affecting more than four million adults in Great Britain. Osteoarthritis is blamed for most of this $^{2}$ and the knee joint is a particularly common site to be affected-one in four people aged over 55 years have knee pain ${ }^{3}$ and by the age of 65 years $30 \%$ of men and $40 \%$ of women have radiographic changes of knee osteoarthritis. ${ }^{4}$
Knee osteoarthritis is therefore likely to provide a major contribution to disability in the general population. There are problems, however, with this assumption. Firstly, although radiographic changes are predictive of the later development of disability, ${ }^{5}$ the relation of knee osteoarthritis with current locomotor impairment has never been specifically examined. Secondly, there are reasons to suspect that the correlation between radiographic severity and level of disability may be poor.

Traditionally the severity of osteoarthritis has been assessed using a system which scores radiographic features believed to be characteristic of this disorder. ${ }^{6}$ Concordance of radiographic osteoarthritis with pain and clinical signs is poor, however, particularly at the knee. ${ }^{7}$ Problems with the scoring system (for example, not including patellofemoral views ${ }^{9}$ ) may account for some of these difficulties, but this does not appear to be the whole story as some work suggests that many diverse factors may be important correlates of pain in osteoarthritis. ${ }^{10}$

Although pain cannot be assumed to be synonymous with disability, the discordance between symptoms and radiographic appearances suggests that there may be determinants of functional impairment in this disorder other than radiographic severity. One important contender is quadriceps femoris weakness: the association of thigh muscle wasting with knee osteoarthritis has been recognised since its earliest descriptions ${ }^{11}$ and treatment traditionally includes exercises intended specifically to increase quadriceps strength. ${ }^{12}$ Research has elucidated mechanisms for an interaction between knee damage and quadriceps dysfunction ${ }^{13}$ which may be particularly adverse in the context of the profound reductions in muscle mass and strength which already occur with increasing age. ${ }^{14}$

To clarify some of these issues we have therefore investigated the relations of a number of putative determinants of disability including radiographic score and quadriceps strength

\section{Subjects and methods}

SUBJECTS

Recruitment was based on respondents to a postal questionnaire asking about knee pain and disability which was sent to all 2101 men and women aged over 55 registered at a general practice in Bristol. It received a response rate of $81 \%$ and has been described elsewhere. ${ }^{3}$ 
Respondents with knee pain and an equal number of controls were contacted in random order by telephone and invited to participate in the study. Cases and controls were matched by gender and were born within 12 months of each other.

KNEE PAIN

The question about knee pain was taken verbatim from the Health and Nutrition Examination Survey ${ }^{15}$ which asks: (a) have you ever had pain in or around a knee on most days for at least a month? (b) if so, have you had any knee pain during the last year?

An affirmative response to the two parts of the question was required for a subject to be designated 'knee pain positive'. For the purposes of analysis a score was derived from this in which $0=$ no pain, $1=$ pain in one knee, and $2=$ pain in two knees.

\section{DISABILITY}

The measure of disability used in this analysis was the total score provided by the Stanford Health Assessment Questionnaire modified for use in this country. ${ }^{16}$ Three parts of the questionnaire (sections 2,4 , and 8 ) are related to lower limb function and were taken together as a 'lower limb score' (range 0-9) for the analysis.

ASSESSMENT OF QUADRICEPS FUNCTION

Quadriceps femoris isometric strength was measured using a system based on the established method of Edwards et al, ${ }^{17}$ which has been shown to be reliable and reproducible. ${ }^{1819}$ In our study we used a converted couch rather than a chair. The subject lay supine with the knee flexed at $90^{\circ}$ over the end of the couch. A foot harness was attached via an inextensible wire to an electronic strain gauge (UF2 $45 \mathrm{~kg}$ strain gauge transducer, Pioden Controls Ltd, Canterbury, United Kingdom) which relayed a signal to a BBC computer. This voluntary effort was displayed as a force $v$ time graph and a cursor provided a measurement of maximum voluntary contraction, defined as the maximum force sustained for at least one second. The best of three recordings was taken, and if there was a large discrepancy between the last two readings, a fourth measurement was obtained. The mean of the right and left maximum voluntary contractions was used for analysis.
RADIOGRAPHY

Weightbearing anteroposterior knee radiographs and recumbent laterals at $30^{\circ}$ of flexion were performed using a standardised technique.

To reflect the variability in compartmental predilection and the bilaterality of knee osteoarthritis, and to optimise reproducibility, a scoring system based on the central three features of the Kellgren and Lawrence scale was used. $^{6}$ Joint space narrowing (0-2), osteophytosis (0-2), and sclerosis $(0-1)$ were scored in the medial, lateral, and patellofemoral compartments of each knee. This provided a maximum score of 5 for each compartment, and the scores from each of the six compartments were summated to provide an overall value for each subject ranging from 0 to 30 .

To assess the repeatability of this system we selected 100 knee radiographs representing the spectrum of disease severity from normal to severe. These were then scored twice in a blinded fashion by a single trained observer (TEM). Repeatability was assessed using the $\kappa$ statistic.

\section{STATISTICAL METHODS}

The distributions of age, knee pain, disability, and radiographic knee osteoarthritis in the study subsamples were analysed using a $\chi^{2}$ test. The relations between variables were investigated first using simple linear regression for continuous variables (age and quadriceps strength) and Spearman rank correlation for discontinuous measures. The relations of quadriceps strength and radiographic score were also investigated using a proportionate analysis based on a $\chi^{2}$ test, or analysis of variance as described by Gardner and Altman. ${ }^{20}$ The determinants of disability were then explored using a logistic regression model.

\section{Results}

SUBJECTS

Complete data were obtained on the first 159 from a total of 513 subjects who attended (table 1). These 159 subjects were younger (mean age 70.2 years $v 72.4 ; \mathrm{p}<0.05$ ) and less disabled $\left(\chi^{2}=11 \cdot 2\right.$; one degree of freedom; $p<0.05)$ than the remainder. The response rate to the telephone invitation was about $80 \%$, though this remains necessarily an estimate because a number were unobtainable and clear

Table 1 Clinical data for 159 subjects responding to questionnaire

\begin{tabular}{|c|c|c|c|c|}
\hline & \multicolumn{2}{|l|}{ Men } & \multicolumn{2}{|l|}{ Women } \\
\hline & $\begin{array}{l}\text { Knee pain negative } \\
(n=36)\end{array}$ & $\begin{array}{l}\text { Knee pain positive } \\
(n=34)\end{array}$ & $\begin{array}{l}\text { Knee pain negative } \\
(n=53)\end{array}$ & $\begin{array}{l}\text { Knee pain positive } \\
(n=36)\end{array}$ \\
\hline Mean (SD) age (years) & $72 \cdot 6(7 \cdot 5)$ & $71 \cdot 8(4 \cdot 7)$ & $70 \cdot 8(9 \cdot 1)$ & $65 \cdot 4(7 \cdot 5)$ \\
\hline $\begin{array}{l}\text { Mean (SD) quadriceps maximum } \\
\text { voluntary contraction }(\mathrm{kgF})\end{array}$ & $27 \cdot 8(9 \cdot 3)$ & $25 \cdot 8(11 \cdot 5)$ & $16 \cdot 7(5 \cdot 7)$ & $15 \cdot 0(5 \cdot 8)$ \\
\hline Subjects with osteoarthritis (\%) & $13 \cdot 9$ & $82 \cdot 4$ & $33 \cdot 0$ & $69 \cdot 4$ \\
\hline Median radiographic score & 0 & 4 & 0 & 5 \\
\hline Disabled subjects` $(\%)$ & 38.9 & $44 \cdot 1$ & $15 \cdot 1$ & $38 \cdot 9$ \\
\hline
\end{tabular}

^Lower limb Health Assessment Questionnaire score greater than 0 . 
Table 2 Pearson's correlation coefficients for age, quadriceps strength (maximum voluntary contraction), radiographic osteoarthritis severity score, knee pain score, and disability score (lower limb sections of the Stanford Health Assessment Questionnaire)

\begin{tabular}{|c|c|c|c|c|}
\hline & $\begin{array}{l}\text { Quadriceps } \\
\text { strength }\end{array}$ & $\begin{array}{l}\text { Radiographic } \\
\text { score }\end{array}$ & Knee pain & Disability \\
\hline Age & $\begin{array}{l}-0.50 \text { (women) } \\
-0.26 \text { (meñ) }\end{array}$ & $0 \cdot 21$ & NS & NS \\
\hline $\begin{array}{l}\text { Quadriceps strength } \\
\text { Radiographic score } \\
\text { Knee pain }\end{array}$ & & $-0 \cdot 21$ & $\begin{array}{l}\text { NS } \\
0 \cdot 43\end{array}$ & $\begin{array}{r}-0 \cdot 35 \\
0 \cdot 24 \\
0 \cdot 32\end{array}$ \\
\hline
\end{tabular}

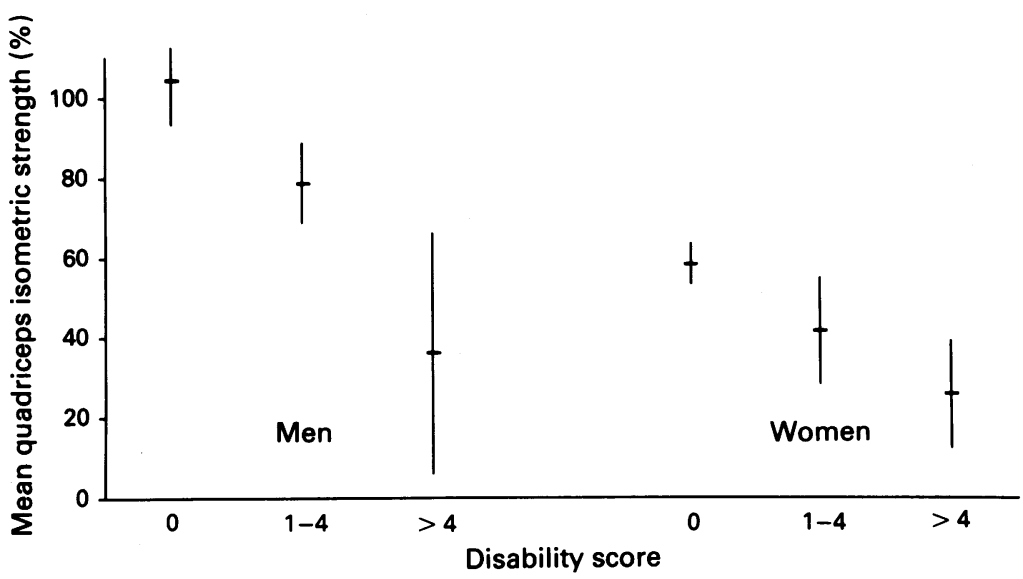

Figure 1 Mean quadriceps isometric strength corrected for age at three levels of disability (lines indicate $95 \%$ confidence limits).

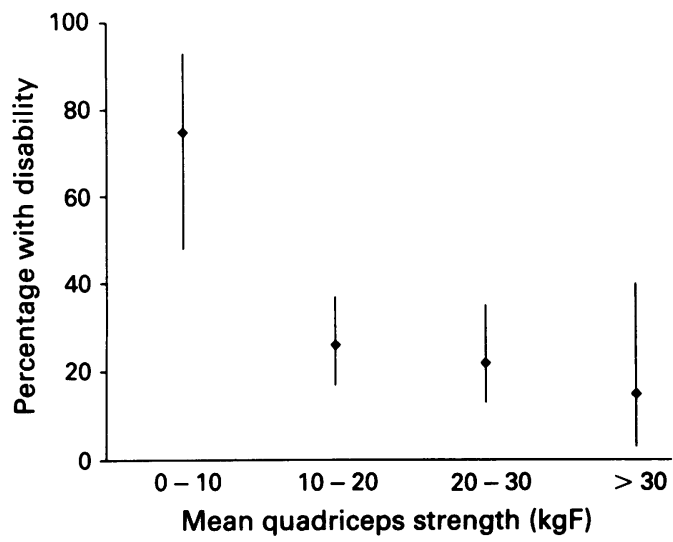

Figure 2 Percentage of reported disability (lower limb Health Assessment Questionnaire score greater than 0) in subjects with increasing increments of quadriceps strength (lines indicate $95 \%$ confidence limits).

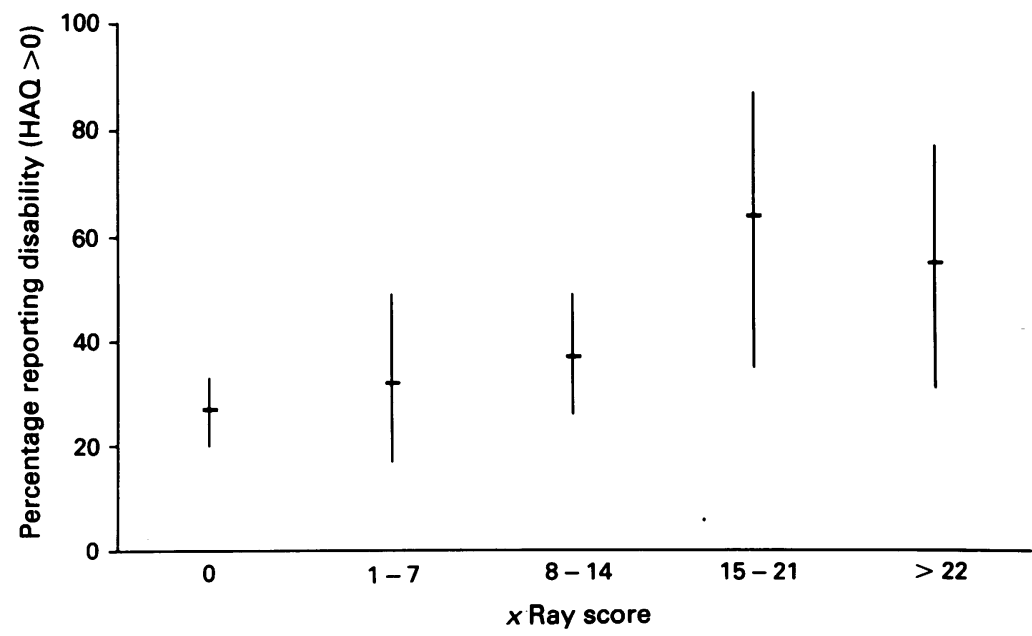

Figure 3 Percentage of subjects reporting disability (lower limb Health Assessment Questionnaire score greater than 0) with increasing severity of radiographic knee osteoarthritis (lines indicate $95 \%$ confidence intervals). records of the large number of calls made were difficult to keep. The data suggest, however, that there was a bias in favour of younger and fitter subjects attending.

\section{CORRELATIONS}

Table 2 gives the correlation matrix between the variables in the subjects tested. For all variables higher correlations were provided by linear regression analysis than by rank correlation. Quadriceps strength was more closely correlated with age in women than men but other correlations did not relate to gender.

QUADRICEPS STRENGTH AND DISABILITY

A clear inverse relation between disability and quadriceps strength remained after adjustment for age using the coefficient derived from linear regression of mean quadriceps maximum voluntary contraction against age in subjects with normal knees (fig 1). Subjects with a measured quadriceps strength of less than 10 $\mathrm{kgF}$ were more likely to report disability, but differences between higher increments of quadriceps strength were not significant, suggesting a threshold effect (fig 2).

RADIOGRAPHIC SCORE AND DISABILITY

The $\kappa$ statistic for radiographic repeatability derived from the score compressed into a three point scale was 0.57 . Figure 3 shows radiographic changes of knee osteoarthritis in relation to levels of disability. The frequency of reported disability (lower limb Health Assessment Questionnaire score greater than 0 ) among subjects with radiographic scores of $\geqslant 15$ was significantly higher than normal subjects $(p<0 \cdot 05)$, but differences were not apparent below this score, again suggesting a threshold effect.

REGRESSION MODELS

Logistic regression was undertaken because of the non-normal distribution of the Health Assessment Questionnaire score, and because of a possibility that linear regression might favour a continuous dependent variable such as quadriceps strength in favour of a discontinuous measure such as radiographic score. Despite this, when added to the model as four categories, radiographic score did not show any significant effect $(p>0 \cdot 1)$. Presenting quadriceps strength as a continuous variable or as two categories made little difference to these results, nor did confining the analysis to those with radiographic changes (score greater than 0 ). Table 3 gives the odds ratios (odds ratio $=$ probability $/ 1-$ probability) and their $95 \%$ confidence limits.

\section{Discussion}

The central conclusion of this study is that, of the variables studied, three exert important independent effects on functional ability in the age group investigated: quadriceps weakness, 
Table 3 Logistic regression analysis of quadriceps strength (as continuous variable), knee pain, sex, age, and radiographic score as explainants of variation in lower limb Health Assessment Questionnaire score in the whole study group and confined to those with radiographic osteoarthritis of the knee

\begin{tabular}{|c|c|c|c|c|}
\hline & \multicolumn{2}{|l|}{ Whole group $(n=159)$} & \multicolumn{2}{|c|}{ Subgroup with osteoarthritis $(n=98)$} \\
\hline & Odds ratio & $95 \%$ confidence limit & Odds ratio & $95 \%$ confidence limit \\
\hline $\begin{array}{l}\text { Quadriceps strength } \\
\text { Knee pain }\end{array}$ & $0.84($ per $\mathrm{kgF})$ & 0.77 to 0.91 & $0 \cdot 84$ & 0.77 to 0.92 \\
\hline One knee & 1.67 ( $v$ no pain) & $1 \cdot 8$ to $15 \cdot 4$ & $3 \cdot 41$ & 0.84 to 13.71 \\
\hline Two knees & 1.48 ( $v$ no pain) & 1.5 to 12.4 & 1.72 & 0.47 to 6.48 \\
\hline Age & 1.06 (per year) & $1 \cdot 0$ to $1 \cdot 12$ & $1 \cdot 04$ & 0.97 to 1.11 \\
\hline Gender & 0.38 (men $v$ women) & 0.13 to $1 \cdot 12$ & 0.43 & 0.11 to 1.61 \\
\hline
\end{tabular}

the presence of knee pain, and increasing age. Of these, quadriceps weakness appears to be the most important, whereas radiographic score itself makes no significant independent contribution to disability.

The odds ratios give some idea of the magnitude of the impact of these variables in terms of reported disability. For example, the risk of disability decreases by 0.88 with each $1 \mathrm{kgF}$ increment in quadriceps strength. Therefore a subject with a mean quadriceps strength of $20 \mathrm{kgF}$ compared with another with $30 \mathrm{kgF}$ will therefore have an increased risk of disability of $(1 / 0 \cdot 84)^{10}=5 \cdot 7$. Knee pain confers a risk of approximately 1.5 compared with no pain, and an increment of 20 years of age (within the older than 55 years age group) confers a risk of disability of about $1 \cdot 8$.

Further insight into the interplay between quadriceps function and locomotor impairment is provided by the threefold increase in frequency of reported disability in subjects with quadriceps strength less than $10 \mathrm{kgF}$. This finding suggests some form of threshold effect, and is in keeping with the concept of a critical strength below which certain activities of daily life become impossible, as suggested by Young. ${ }^{13}$

Interaction may also exist between muscle strength as measured by a voluntary contraction and psychological factors. Summers et al showed an association between psychometric variables and perceived levels of pain and disability. ${ }^{10}$ It is possible that similar factors might influence maximum effort during strength testing which could enhance a correlation between weakness and self reported disability.

The absence of any detectable independent effect of radiographic osteoarthritis on functional ability supports the original hypothesis, but is, perhaps, surprising. One possible explanation relates to the lack of validity of the radiographic scoring system. Early research using the Kellgren and Lawrence radiographic osteoarthritis score showed relatively poor reproducibility, ${ }^{21}$ but in later studies its performance has been improved by assigning strict verbal criteria to each grade based on the original radiographic features. ${ }^{22}$ For this reason, and to reflect the heterogeneity of knee osteoarthritis in terms of compartmental involvement and bilaterality, each of the six compartments were scored independently for joint space narrowing, osteophytosis, and sclerosis. These scores were then summated to provide an overall grade for each subject which proved adequately repeatable in this study. It is possible that this methodology provided a non-representative reflection of severity, though this seems unlikely in the context of current concepts of osteoarthritis. Alternatively, the problem may relate to the scored radiographic features as indicators of the osteoarthritic process itself. Osteoarthritis is a disease defined by a constellation of histological features, but in epidemiological surveys the radiographic score has provided the diagnostic gold standard. ${ }^{4-7} 1522$ The relation between radiographic changes of knee osteoarthritis and symptoms is known to be poor $^{722}$ and certain features which contribute to the radiographic diagnosis such as osteophytosis may relate more to ageing than any disease. ${ }^{23}$ Some recent work has also shown that the predictive value of joint space narrowing for cartilage loss is suboptimal in individuals, though the degree of correlation is at a level acceptable for epidemiological study. ${ }^{24}$ In our analysis, similar results were generated by the use of a joint space narrowing, osteophyte, or sclerosis score, suggesting little difference in validity between them.

The most plausible explanation for the findings of the regression model is that pain and muscle weakness are the mechanisms through which osteoarthritis exerts an influence on functional ability, and the correlations of radiographic score with knee pain and quadriceps strength agree with this. Alternative hypotheses are that radiographic features reflect outcome and tell us little about the disease process, or, more provocatively, that the radiopathological changes believed to constitute osteoarthritis do not represent a disease entity and are not a cause of disability.

Finally, disability is a concept, measurement of which has philosophical was well as practical problems. Interest in this field over the last decade has generated validated functional assessment instruments such as the Health Assessment Questionnaire, without which a study such as this would not have been possible. The Health Assessment Questionnaire itself has been extensively validated in subjects with rheumatic diseases and correlations between reported and observed performance range from 0.47 to 0.88 for individual questions with higher values obtained for aggregated scores. ${ }^{25} 26$ Work using this instrument showing greater levels of disability in women has raised a question about liability to female bias $^{27}$ and it is therefore interesting that, though the community survey 
also showed an excess of disability in women, the regression analysis found no significant independent effect of gender. This lends further weight to the suggestion that women are more likely to experience functional limitation than men.

The general applicability of the findings of this study may be reduced by biases in patient selection. As radiographic changes of osteoarthritis are well recognised to occur in the absence of symptomatology it was necessary to examine a knee pain negative group as well as symptomatic subjects. The selection method used represented a practical compromise between seeing a random population sample (which would have resulted in a low yield of cases) and using hospital outpatients (which would have resulted in unacceptable selection bias).

The study sample, though contacted in random order, cannot be assumed to be representative of the study population and it is likely that biases in favour of healthier and fitter subjects attending for assessment were operative. This effect would be particularly important if either the prevalence of radiographic change was considerably less in the tested group, or if the relation between muscle strength and disability was decreased in the face of more advanced osteoarthritis. Neither of these, however, appears to be so; subjects with disability and with radiographic knee osteoarthritis were well represented in the study group ( 32 and $48 \%$ respectively), and the logistic regression analysis confined to the subgroup with knee osteoarthritis provided identical results.

The public health implications of this study are therefore not confined to those with osteoarthritis alone. Decline in muscle function after about 55 years of age is an apparently almost universal phenomenon 181928 and significant negative correlations between age and quadriceps maximum voluntary contraction were found in this sample. The causes of this decrease in muscle function are not fully understood but it has been suggested that a large proportion is likely to be due to inactivity. ${ }^{29}$ Much of this may be reversible: improvements in muscle function have been documented in elderly volunteers participating in exercise programmes and the benefits appear to be substantial. ${ }^{29}$ Applied to the general population, strategies designed to optimise muscle strength may have the potential to reduce a vast burden of disability, dependency, and cost.

We thank Shelagh Snow, research sister, Dr Rosie Jones, muscle physiologist, Dr Alan Preece, chief medical physicist, Bristol Royal Infirmary, Mr Nick Taube, statistician, St Thomas's Hospital, and Dr Tim Paine and patients of the Whiteladies Road Health Centre, Bristol. The project was funded by the Arthritis and Rheumatism Council.

1 Martin J, Meltzer H, Eliot D. The prevalence of disability among adults. London: Office of Population Censuses and Surveys, Social Survey Division, HMSO, 1988.
2 Badley E M, Thompson R P, Wood P H N. The prevalence and severity of major disabling conditions. Int $\mathcal{f}$ Epidemiol $1978 ; 7: 145-51$

3 McAlindon T E, Cooper C, Kirwan J R, Dieppe P A. Knee pain and disability in the community. $\mathrm{Br} \mathcal{f}$ Rheumatol 1992; 31: 189-92.

4 Van Saase J L C M, Van Romunde L K J, Cats A, Vandenbroucke J P, Valkenberg H A. Epidemiology of osteoarthritis: Zoetermeer survey. Comparison of radiological osteoarthritis in a Dutch population with that in 10 other populations. Ann Rheum Dis 1989; 48: 271-80.

5 Hochberg M C, Lawrence R C, Everett D F, CornoniHuntley J. Epidemiologic associations of pain in osteoarthritis of the knee: data from the National Health and Nutrition Examination Survey and from the National Health and Nutrition Examination-1 epidemiologic follow-up survey. Semin Arthritis Rheum 1989; 4 (suppl 2): 4-9.

6 Kellgren J H, Lawrence J S. The epidemiology of chronic rheumatism: atlas of standard radiographs of arthritis. Oxford: Blackwell Scientific, 1963.

7 Lawrence J S, Bremner J M, Bier F. Osteoarthrosis: prevalence in the population and relationship between symptoms and x-ray changes. Ann Rheum Dis 1966; 25: $1-23$.

8 Claessens A A M, Schouten J S A G, Van den Ouweland F A, Valkenburg H A. Do clinical findings associate with radiographic osteoarthritis of the knee? Ann Rheum Dis 1990; 49: 771-4.

9 McAlindon T E, Snow S, Cooper C, Dieppe P A Radiographic patterns of osteoarthritis of the knee join in the community: the importance of the patellofemora joint. Ann Rheum Dis 1992; 51: 844-9.

10 Summers M N, Haley W E, Reveille J D, Alarcon G S. Radiographic assessment and psychological variables as predictors of pain and functional impairment in predictors of pain and functional impairment in
osteoarthritis of the knee or hip. Arthritis Rheum 1988; 31: 204-9.

11 Adams R. A treatise on rheumatic gout or chronic rheumatic arthritis of all the joints. London: Churchill, 1857

12 Rudd E. Physiatric management of osteoarthritis. Clin Rheum Dis 1985; 2: 433-5.

13 Young A. Exercise physiology in geriatric practice. Acta Med Scand Suppl 1986; 711: 227-32.

14 Aniansson A, Sperling L, Rundgren A, Lehnberg E. Muscle function in 75 year old men and women: a longitudinal study. Scand $\mathcal{F}$ Rehabil Med Suppl 1983; 9: 92-102.

15 Maurer K. Basic data on arthritis of the hip, knee and sacroiliac joints in adults ages 25-74, United States 1971-1975 vital and health statistics, series 11, no 213 . Hyatsville, MD: National Center for Health Statistics; 1979. DHEW Publication No.: (PUS) 79-1661.

16 Kirwan J R, Reeback J S. Stanford Health Assessment Questionnaire modified to assess disability in British Questionnaire modified to assess disability in British patients with $206-9$.

17 Edwards R H T, Young A, Hosking G P, Jones D A. Human skeletal muscle function: description of tests and normal values. Clin Sci Mol Med 1977; 52: 283-90.

18 Young A, Stokes $M$, Crowe M. Size and strength of the quadriceps muscles of old and young women. Eur $\mathcal{f}$ Clin Invest 1984; 14: 282-7.

19 Young A, Stokes $M$, Crowe $M$. Size and strength of the quadriceps muscles of old and young men. Clin Physiol 1985; 5: 145-54.

20 Gardner M J, Altman D G. Calculating confidence intervals for proportions and their differences. In: Gardner M J for proportions and their differences. In: Gardner M Altman D G, eds. Statistics

21 Kellgren J H, Lawrence J S. Radiological assessment of osteo-arthrosis. Ann Rheum Dis 1957; 16: 494-501.

22 Felson D T, Naimark A, Anderson J, et al. The prevalence of knee osteoarthritis in the elderly: the Framingham osteoarthritis study. Arthritis Rheum 1987; 30: 914-8.

23 Hernborg J, Nilsson B E. Age and sex incidence of osteophytes in the knee joint. Acta Orthop Scand 1973; 44 66-8.

24 Fife R S, Brandt K D, Braunstein E M, et al. Relationship between arthroscopic evidence of cartilage damage an radiographic evidence of joint space narrowing in early osteoarthritis of the knee. Arthritis Rheum 1991; 34: 377-82.

25 Fries J F, Spitz P, Kraines G, Holman H R. Measuremen of patient outcome in arthritis. Arthritis Rheum 1980; 23 of patient $137-45$.

26 Fries J, Spitz P, Young D. The dimensions of health outcomes: the Health Assessment Questionnaire, outcomes. the Health Assessment Questionnaire, disability and pain scales. F Rheumatol 1982; 9: 789-93.

27 Thompson P W, Pegley F S. A comparison of disability measured by the Stanford Health Assessmen Questionnaire disability scales in male and female

28 Asmussen E, Fruensgaard K, Norgaard S. A follow-up survey of selected physiologic functions in former physica education students after forty years. $\mathcal{F} \mathrm{Am}$ Geriatr So 1975; 23: 442-50.

29 Bassey E J. Benefits of exercise in the elderly. In: Isaacs B ed. Recent advances in geriatric medicine, No 3 . London: Churchill Livingstone, 1985. 